\title{
Efeito da cafeína sobre a performance no treinamento intervalado de alta intensidade
}

\author{
Effect of caffeine on High Intensity Interval Training performance \\ Efecto de la cafeína sobre el rendimiento en el entrenamiento por intervalos de alta intensidad
}

Recebido: 21/06/2021 | Revisado: 29/06/2021 | Aceito: 03/07/2021 | Publicado: 14/07/2021

Matheus Alexandre da Silva Machado
ORCID: https://orcid.org/0000-0003-3510-8479
Universidade do Estado do Rio de Janeiro, Brasil
E-mail: matheusam45@gmail.com
Alexandre Fernandes Machado
ORCID: https://orcid.org/0000-0002-6728-9107
Universidade do Estado do Rio de Janeiro, Brasil
E-mail: xdmachado@ gmail.com
Andréia da Silva Fernandes Campos
ORCID: https://orcid.org/0000-0002-5258-8139
Universidade do Estado do Rio de Janeiro, Brasil
E-mail: andreiadasilvaf@ gmail.com
Israel Felzenszwalb
ORCID: https://orcid.org/0000-0003-1677-197X
Universidade do Estado do Rio de Janeiro, Brasil
E-mail: uerj.felzen@ gmail.com

\section{Resumo}

No presente estudo, foram investigados através de uma revisão sistemática, os efeitos da utilização de cafeína sobre o indivíduo nas sessões de HIIT. A seleção de artigos foi realizada nas bases de dados Medline/PubMed, Science Direct, Scholar Google e Scielo, e encontraram-se 2301 estudos. Após aplicar os critérios de inclusão e exclusão foram considerados elegíveis apenas cinco artigos para a revisão sistemática. Entre esses estudos, o total de participantes foi de 80 indivíduos, sendo 24 mulheres e 56 homens, com idades entre 18 e 30 anos. Os protocolos utilizados consistiram em dois tipos: agudos e crônico, avaliando parâmetros antropométricos e/ou metabólicos. Nos experimentos que utilizaram uma abordagem crônica, foram encontrados resultados favoráveis à perda de gordura corporal e melhora de índices metabólicos. Por outro lado, os estudos com abordagem aguda observaram melhora na performance através do retardo da fadiga muscular, embora, sem diferença significativa de massa corporal ao final da sessão. Ainda, um dos estudos com intervenção crônica não observou diferença significativa entre os grupos. A cafeína associada ao HIIT é capaz de trazer benefícios ao indivíduo, independentemente de seu condicionamento físico. Entretanto, ainda é preciso mais estudos para um melhor entendimento da extensão e condição desses benefícios.

Palavras-chave: Cafeína; Exercício físico; Treinamento intervalado de alta intensidade.

\begin{abstract}
The aim of the present study was to investigate, through a systematic review, the effects of caffeine use on the individual in HIIT sessions. After selecting articles in the Medline / PubMed, Science Direct, scholar google and Scielo databases, 2301 studies were found. After applying the inclusion and exclusion criteria, only five articles were considered eligible for systematic review. Among these studies, the total number of participants was 80 individuals, 24 women and 56 men, aged between 18 and 30 years. The protocols used consisted of two types: acute and chronic, assessing anthropometric and / or metabolic parameters. In experiments that used a chronic approach, results were found favorable to the loss of body fat and improvement of metabolic indexes. On the other hand, studies with an acute approach observed an improvement in performance through the delay of muscle fatigue, however, without significant difference in body mass at the end of the session. Still, one of the studies with chronic intervention did not observe a significant difference between the groups. Caffeine associated with HIIT can bring benefits to the individual, regardless of their physical condition. However, further studies are needed to better understand the extent and condition of these benefits.
\end{abstract}

Keywords: Caffeine; Physical exercise; High intensity interval training.

\section{Resumen}

En el presente estudio, se investigaron los efectos del uso de cafeína en el individuo en sesiones de HIIT a través de una revisión sistemática. La selección de artículos se realizó en las bases de datos Medline / PubMed, Science Direct, Scholar Google y Scielo, y se encontraron 2301 estudios. Después de aplicar los criterios de inclusión y exclusión, 
solo cinco artículos se consideraron elegibles para la revisión sistemática. Entre estos estudios, el número total de participantes fue de 80 personas, 24 mujeres y 56 hombres, con edades comprendidas entre los 18 y los 30 años. Los protocolos utilizados fueron de dos tipos: agudos y crónicos, evaluando parámetros antropométricos y / o metabólicos. En experimentos que utilizaron un enfoque crónico, se encontraron resultados favorables para la pérdida de grasa corporal y la mejora de los índices metabólicos. Por otro lado, los estudios con abordaje agudo observaron una mejora en el rendimiento a través del retraso de la fatiga muscular, aunque sin diferencia significativa en la masa corporal al final de la sesión. Aún así, uno de los estudios con intervención crónica no encontró diferencias significativas entre los grupos. La cafeína asociada con el HIIT puede brindar beneficios a las personas, independientemente de su condición física. Sin embargo, se necesitan más estudios para comprender mejor el alcance y la condición de estos beneficios.

Palabras clave: Cafeína; Ejercicio físico; Entrenamiento por intervalos de alta intensidad.

\section{Introdução}

A cafeína é uma substância química psicotrópica muito consumida no mundo (Embrapa, 2019), seja através de alimentos como café ou com uso de suplementos para exercício físico, chamados pré-treino (Gomes et al. 2014).

Seu uso como estimulante é amplamente explorado e conhecido, funcionando como antagonista competitivo dos receptores adrenérgicos, o que causa aumento da atividade neuronal e mudanças fisiológicas decorrentes da ligação da cafeína com os receptores de adenosina, como liberação de norepinefrina, serotonina e aumento de catecolaminas circulantes (Nehlig, 2016). Alterações provocadas pela cafeína aumentam o estado de alerta e excitação do indivíduo, trazendo maior disposição para a prática de exercícios (Santos et al. 2015). Entretanto, o uso crônico da cafeína está associado a diminuição de catecolaminas circulantes, estímulo da lipólise e melhora de índices de glicemia e insulinemia, sem perda da ação estimulante (Braga et al. 2000).

Devido ao seu efeito estimulante e às mudanças fisiológicas causadas (Nehlig, 2016), a cafeína vem sendo utilizada em diversas modalidades esportivas como um recurso ergogênico (Braga et al. 2000), inclusive no HIIT, que é uma modalidade que tem alcançado cada vez mais popularidade nos últimos anos (Boullosa, 2019; Machado et al. 2018; Machado, 2017).

O treinamento intervalado de alta intensidade (HIIT) consiste em sessões com estímulos de alta intensidade seguidos de um período de recuperação, que pode ser passiva (parado) ou ativa (em movimento), o tempo total das sessões podem variar entre 4 e 30 minutos (Machado et al. 2018).

O HIIT ganhou muito destaque nos últimos anos pela sua versatilidade, facilidade de aplicação e curta duração da sessão de treino (Machado, 2017). Dependendo da sua aplicação, pode receber alguns nomes diferentes, também conhecidos como SIT, HIT, HIRT, HIFT, HBW (Boullosa, 2019; Machado et al. 2018), mas todos possuem o mesmo princípio: um intervalo de alta intensidade seguido de um intervalo de recuperação e assim alternadamente. Outro ponto importante que ajudou na popularização, foram as evidências de redução de gordura corporal (Mundt \& Bertolini, 2016; Belmiro \& Navarro, 2016; Sousa, 2019).

Considerando o uso da cafeína como conhecido estimulante, o objetivo do presente estudo foi investigar, através de uma revisão sistemática, os efeitos da utilização de cafeína sobre o indivíduo nas sessões de HIIT.

\section{Metodologia}

O estudo teve sua base metodológica fundamentada em Collado et al. (2013), sendo um estudo quantitativo. A busca eletrônica foi realizada através das seguintes bases de dados: Medline/PubMed, Science Direct, scholar google e Scielo. Os termos selecionados como descritores de busca foram: "caffeine" e "high intensity interval training", "high intensity interval", "interval training" e "high intensity exercise". A busca foi realizada entre 01 de outubro e 02 de novembro de 2020. 
Os artigos foram selecionados de forma que atendessem aos critérios de inclusão: palavra-chave no título e no resumo, protocolo de intervenção que utilize HIIT e data de publicação entre janeiro de 2015 e agosto de 2020. Como critério de exclusão foram utilizados os seguintes critérios: estudos realizados com animais, artigos de revisão, dissertação e tese.

$\mathrm{Na}$ etapa de identificação, 32 artigos foram selecionados com os termos "caffeine" e "high intensity interval training", "high intensity interval", "interval training" ou "high intensity exercise". Após a aplicação dos critérios de inclusão e exclusão estabelecidos, foram contemplados 5 estudos (Figura 1).

Figura 1: Fluxograma com as fases da metodologia de seleção dos artigos incluídos na revisão sistemática.
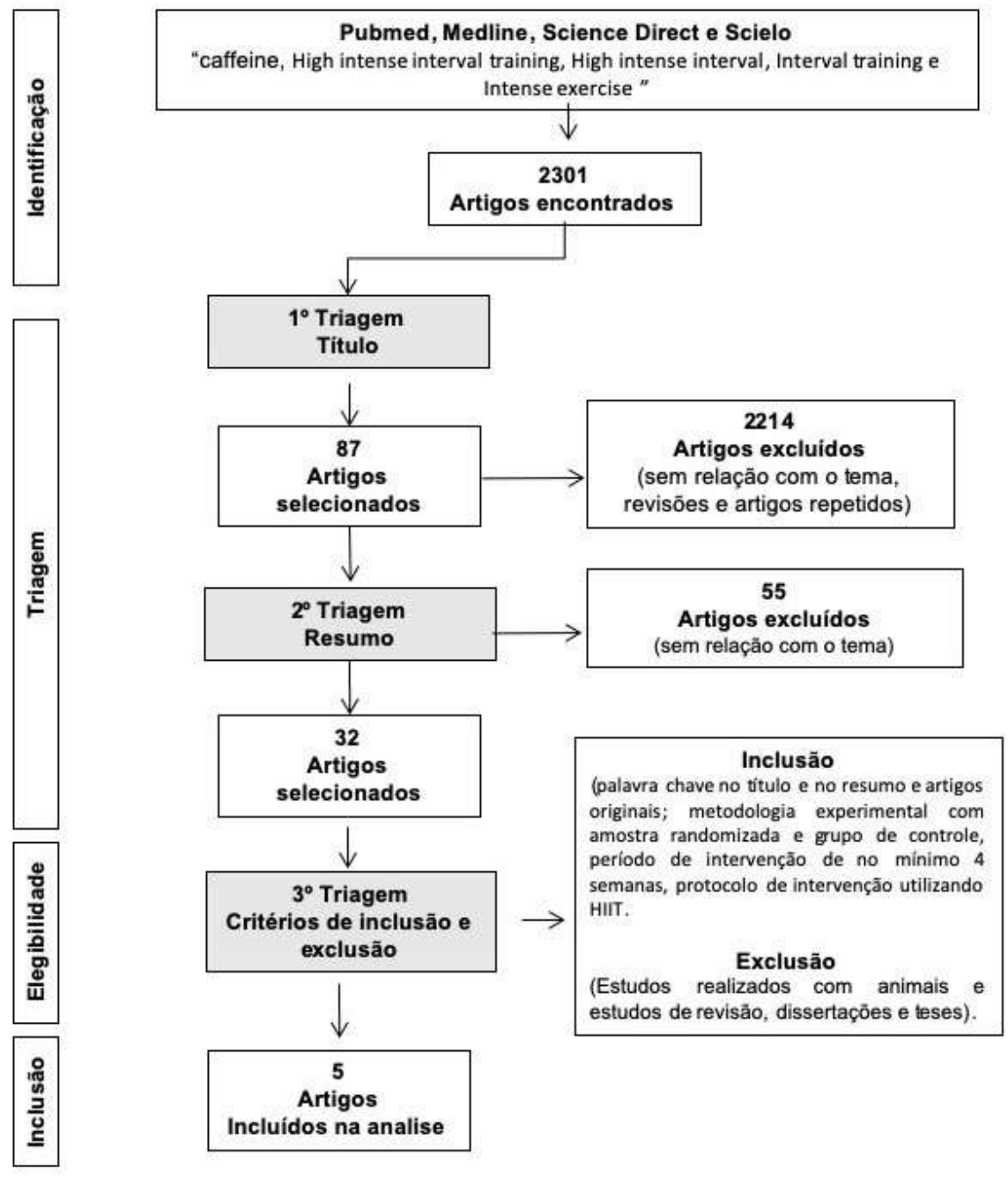

Fonte: Dados da pesquisa bibliográfica.

\section{Resultados}

O total de participantes nos estudos selecionados foi de 80 indivíduos, sendo 24 mulheres e 56 homens, com idades entre 18 e 30 anos (Tabela 1). As modalidades utilizadas nos estudos foram bicicleta e corrida ambos em ergômetros. Nos estudos selecionados, dois avaliaram a resposta aguda da cafeína sobre a performance e três avaliaram a resposta crônica. 
Tabela 1. Periódicos publicados, perfil da amostra, desenho experimental e desfecho dos experimentos.

\begin{tabular}{|c|c|c|c|c|c|}
\hline & $\begin{array}{l}\text { Alkhatib et al. } \\
2020\end{array}$ & $\begin{array}{c}\text { Ferreira, G.A. et al. } \\
2019\end{array}$ & $\begin{array}{l}\text { Ferreira, G.A. et } \\
\text { al. } 2018\end{array}$ & $\begin{array}{c}\text { Devenney, Simon et } \\
\text { al. } 2017\end{array}$ & $\begin{array}{c}\text { Kasper, Andreas M } \\
\text { et al. } 2016\end{array}$ \\
\hline Periódico & $\begin{array}{l}\text { Medicine and } \\
\text { Science in Sports } \\
\text { and Exercise }\end{array}$ & $\begin{array}{l}\text { Brazilian Journal of } \\
\text { Medical and } \\
\text { Biological Research }\end{array}$ & $\begin{array}{l}\text { Frontiers in } \\
\text { Physiology }\end{array}$ & $\begin{array}{l}\text { Applied physiology, } \\
\text { nutrition, and } \\
\text { metabolism }\end{array}$ & $\begin{array}{l}\text { European College of } \\
\text { Sport Science }\end{array}$ \\
\hline Amostra & $\begin{array}{l}24 \text { mulheres, } 21.7 \\
\pm 0.8 \text { anos }\end{array}$ & $\begin{array}{l}20 \text { homens, } 25.9 \pm \\
5.1 \text { anos }\end{array}$ & $\begin{array}{l}20 \text { participantes, } \\
25.9 \pm 5.1 \text { anos }\end{array}$ & $\begin{array}{l}8 \text { homens, } 23 \pm 3 \\
\text { anos }\end{array}$ & 8 homens, $23 \pm 3$ anos \\
\hline $\begin{array}{l}\text { Desenho } \\
\text { experimental }\end{array}$ & $\begin{array}{l}\text { HIIT(bicicleta): } 3 \\
\text { sessões } \\
\text { semana, por } 8 \\
\text { semanas. } \\
\text { Intensidade } \\
\text { treino em } 90 \% \text { de } \\
\text { FC. }\end{array}$ & $\begin{array}{l}\text { HIIT(bicicleta): } 3 \\
\text { sessões por semana, } \\
\text { por } 2 \text { semanas. } \\
\text { Intensidade all out. }\end{array}$ & $\begin{array}{l}\text { HIIT (bicicleta): } 1 \\
\text { sessão de teste pré } \\
\text { treino (96hs antes } \\
\text { da primeira) }+6 \\
\text { sessões de treino } \\
\text { durante } 2 \text { semanas } \\
\text { com intervalo de } \\
48 \mathrm{hs}+1 \text { sessão pós } \\
\text { treino (após 96hs } \\
\text { da última). }\end{array}$ & $\begin{array}{l}\text { Corrida SS de } 45 \\
\text { min e um protocolo } \\
\text { de corrida HIIT } \\
\text { (90\% da velocidade } \\
\text { máxima da esteira) } \\
\text { até a fadiga, } 3 \\
\text { tentativas. }\end{array}$ & $\begin{array}{l}\text { Corrida SS de } 45 \mathrm{~min} \\
\text { e um protocolo de } \\
\text { corrida HII } \\
\text { minuto a } 80 \% \text { VO2 } \\
\text { máx intercalado com } \\
\text { caminhada de } 1 \\
\text { minuto a } 6 \mathrm{~km} \mathrm{/} \mathrm{h).} 3 \\
\text { tentativas. }\end{array}$ \\
\hline
\end{tabular}

Fonte: Dados dos artigos selecionados na pesquisa.

Na Tabela 1 é possível observar um panorama dos estudos selecionados. É importante observar as diferenças entre eles, os quais podem ser divididos em dois grupos: estudos de abordagem aguda e estudos de abordagem crônica.

Dos estudos agudos, Kasper et al. (2015) analisaram a resposta da performance após uma sessão de HIIT na esteira utilizando como parâmetro o tempo total de exercício em três diferentes grupos: grupo 1 (placebo), grupo 2 (enxágue bucal de carboidrato, solução a 10\%) e grupo 3 (enxágue bucal de carboidrato $10 \%$ mais ingestão pré treino de cafeína de 200 mg). Foi observado que o grupo 2 teve uma performance 44,4\% superior e o grupo 3 80,5\% superior ao grupo 1. Quando comparado os resultados do grupo 2 e 3, observou-se que o grupo 3 foi superior ao grupo 2 em $25 \%$.

Devenney et al. (2017) também analisaram a resposta da performance após uma sessão de HIIT de forma aguda, entretanto, utilizou-se como parâmetro a distância total percorrida na sessão. Os participantes foram divididos em três diferentes grupos: grupo 1 (placebo), grupo 2 (enxágue bucal de carboidrato, solução a 10\%) e grupo 3 (enxágue bucal de carboidrato $10 \%$ mais ingestão pré treino de cafeína de $200 \mathrm{mg}$ ), seguindo o mesmo desenho metodológico de Kasper, et al. (2015). Os autores observaram que o grupo 2 obteve uma performance $13 \%$ superior e o grupo 3 , 40,6\% superior ao grupo 1 . Além disso, comparando os grupos 2 e 3, foi observado que o grupo 3 obteve um resultado 24,4\% superior que o grupo 2. Nos estudos que analisaram a resposta aguda (Kasper, 2016; Devenney, 2017), os autores observaram a superioridade do grupo que utilizou a cafeína no experimento.

Recentemente, Alkhatib et al. (2020) observaram os efeitos da cafeína associada ao um programa de oito semanas de HIIT em bicicleta sobre parâmetros metabólicos, composição corporal, capacidade cardiorrespiratória e capacidade aeróbia em mulheres com obesidade. Os 24 voluntários foram divididos em dois grupos: HIIT com cafeína (CAF) e HIIT sem cafeína (PLA), sendo 3 sessões por semana em dias não consecutivos. Foram apresentados os seguintes resultados após as oito 
semanas de treinamento: alteração dos níveis de tolerância à glicose no grupo CAF (-19,1 \%) e PLA (14,5\%); alteração dos níveis de tolerância a insulina no grupo CAF $(0,04 \%)$ e PLA (28,3\%); alteração dos níveis de testosterona de $-16 \%$ em ambos os grupos CAF e PLA e nos níveis de endotoxinas relacionadas à obesidade no grupo PLA (30,8\%) e no grupo CAF $(0,2 \%)$.

Em outro estudo, Ferreira et al. (2019) avaliaram parâmetros de gasto energético e composição corporal antes e após um período de 2 semanas de treinamento HIIT em bicicleta, com frequência de treino de 3 vezes na semana em dias não consecutivos. Durante a sessão de treino não foi observada diferenças significativas no custo energético entre os grupos (CAF e PLA), porém no período de 45 minutos imediatamente após a sessão de treino o custo energético foi superior em 19,7\% no grupo CAF quando comparado do grupo PLA. Embora não haja alteração na massa corporal após o período de treinamento entre os grupos, observou-se uma variação no percentual de gordura corporal de -1,0 no grupo CAF quando comparado ao PLA e uma alteração na soma das dobras cutâneas de $-9,1$ mm no grupo CAF.

Ferreira et al. (2018) observaram os efeitos da cafeína associada a uma sessão de HIIT na bicicleta de forma aguda e crônica sob os parâmetros: interleucina (IL-6 e IL-10), fator de necrose tumoral (TNF-a), creatina quinase (CK) e somente de forma crônica o $\mathrm{VO}_{2}$ Maximo $\left(\mathrm{VO}_{2} \max \right)$ e a potência máxima (Wmax). De forma aguda não houve diferença significativa entre os grupos HIIT sem cafeína (PLA) e HIIT com cafeína (CAF) para IL6, LI-10, TNF-a e CK. Contundo na primeira sessão observou-se um aumento da CK somente para o grupo PLA. Quando comparado os grupos PLA e CAF de forma crônica observou-se uma diminuição IL6, LI-10 e TNF-a e aumento nos níveis de $\mathrm{VO}_{2}$ max e Wmax em ambos os grupos.

\section{Discussão}

O objetivo desse estudo foi investigar, através de uma revisão sistemática, os efeitos da utilização de cafeína associada ao HIIT sobre o indivíduo.

Entre os artigos selecionados, verificamos dois efeitos principais relacionados ao consumo de cafeína no período pré treino: prolongamento do tempo de esforço até o estado de fadiga muscular, observado principalmente nos estudos de abordagem aguda; e perda de gordura corporal com melhora de índices metabólicos, observado nos estudos de abordagem crônica.

Kasper et al. (2015) e Devenney et al. (2017) observaram uma melhora na performance dos atletas, seja correndo uma maior distância ou resistindo por mais tempo ao exercício até a fadiga muscular. Esse resultado está de acordo com estudos anteriores (Mohr et al. 2010), que mostram que a suplementação com cafeína em atividades físicas reduziu as concentrações de potássio no meio extracelular e aumentou sua concentração no meio intracelular, retardando a fadiga muscular.

Além disso, o uso da cafeína está relacionado com uma maior oxidação de ácidos graxos durante a execução de exercícios de alta intensidade, preservando as reservas de glicogênio muscular, o que também retarda a fadiga (Silveira et al. 2015). Entretanto, a abordagem aguda de treino utilizada por Kasper et al. (2015) e Devenney et al. (2017) não foi capaz de provocar diferença significativa nos níveis de gordura corporal, apontando um efeito de médio e longo prazo em relação ao percentual de gordura corporal.

Nos estudos de abordagem crônica, a significativa perda de gordura corporal nos grupos que fizeram uso da cafeína está relacionada com o gasto energético mais elevado no período pós sessão de treino, ou seja, o indivíduo permanece com uma alta taxa de queima calórica por um tempo prolongado após a atividade se comparada com o grupo controle. Esse fato sugere que o emagrecimento é uma consequência do deficit energético causado pelo aumento do gasto energético, o que só poderia ser observado em uma abordagem de treinamento crônico.

Em estudos anteriores (Bhaktha et al. 2015; Conde et al. 2012), o consumo crônico de cafeína foi capaz de reduzir a glicemia e conservar a sensibilidade à insulina em animais e humanos, efeito que foi associado ao não aumento de 
catecolaminas circulantes induzidas por alimentação rica em gorduras e açúcares (Conde et al. 2012) e aumento nas concentrações de adiponectina, que também pode explicar o aumento do gasto energético e da lipólise (Bhaktha et al. 2015).

Sutton-Tyrrell et al. (2005) e Brix-Christensen et al. (2004), mostraram que os índices endotóxicos relacionados à obesidade estão intimamente ligados à resistência à insulina e à glicemia. Alkhatib et al. (2020) observaram uma melhora nos níveis de tolerância à glicose e insulina, o que causaria também a melhora observada nos índices de endotoxicidade relacionada à obesidade.

Entretanto, o estudo de Ferreira G. A. et al. 2018, mostra que os dados da literatura em relação aos efeitos da cafeína ainda são controversos, não encontrando diferenças significativas entre os grupos no treinamento. Porém, a diferença nos marcadores de inflamação após apenas a primeira sessão mostra o efeito citoprotetor da cafeína em indivíduos não habituados ao treino (Santos \& Rocha, 1994). Por outro lado, também mostrou que o HIIT, mesmo em curto prazo, pode gerar respostas adaptativas, como a diminuição de marcadores de inflamação muscular (IL-6, IL-10, TNF-a e CK) após o período de treinamento.

É importante ressaltar que limitações são dispostas nesse artigo, sendo elas o número limitado de estudos sobre o tema na literatura, a não identificação do tipo de metabolização de cafeína dos participantes e ainda a divergência de protocolos adotados nos estudos.

\section{Conclusão}

A cafeína associada ao HIIT traz efeitos benéficos para o indivíduo, seja fisicamente treinado ou em estado de obesidade, podendo ser utilizada como recurso ergogênico e auxiliar na perda de gordura corporal, respeitando os limites diários de dosagem. Contudo, mais estudos são necessários para corroborar os efeitos aqui relatados. Além disso, também há a necessidade de relacionar esses efeitos com a capacidade de metabolização do indivíduo.

Dessa forma, fica como sugestão para estudos posteriores, ensaios que relacionam a capacidade de metabolização e/ou perfil metabólico genético do indivíduo com a dose adequada de cafeína afim de que cada tipo de indivíduo possa alcançar os efeitos benéficos da cafeína de forma apropriada.

\section{Agradecimentos}

Os autores agradecem à Fundação de Amparo à Pesquisa do Estado do Rio de Janeiro - FAPERJ pelo apoio financeiro através da bolsa de iniciação científica.

\section{Referências}

Aguiar, R. A. D., Turnes, T., Cardoso, T. E., Vasconcellos, D. I. C., \& Caputo, F. (2012). Efecto de la ingesta de cafeína en diferentes tareas de tiempo de reacción. Revista Brasileira de Ciências do Esporte, 34(2), 465-476.

Alkhatib, A., Hsieh, M. J., Kuo, C. H., \& Hou, C. W. (2020). Caffeine Optimizes HIIT Benefits on Obesity-associated Metabolic Adversity in Women. Medicine and science in sports and exercise.

Belmiro, W., \& Navarro, A. C. (2016). Os efeitos do treinamento intervalado de alta intensidade para o emagrecimento. RBONE-Revista Brasileira de Obesidade, Nutrição e Emagrecimento, 10(59), 224-230.

Bhaktha, G., Nayak, B. S., Mayya, S., \& Shantaram, M. (2015). Relationship of caffeine with adiponectin and blood sugar levels in subjects with and without diabetes. Journal of clinical and diagnostic research: JCDR, 9(1), BC01.

Boullosa, D. (2019). Muitas palavras para poucos conceitos: O caso do 'HIIT'. Revista Brasileira de Pesquisa em Ciências da Saúde, 5(10), 27-30.

Braga, L. C., \& Alves, M. P. (2008). A cafeína como recurso ergogênico nos exercícios de endurance. Revista Brasileira de Ciência e Movimento, 8(3), 33-38. 
Brix-Christensen, V., Andersen, S. K., Andersen, R., Mengel, A., Dyhr, T., Andersen, N. T., \& Tønnesen, E. (2004). Acute hyperinsulinemia restrains endotoxin-induced systemic inflammatory response: an experimental study in a porcine model. The Journal of the American Society of Anesthesiologists, 100(4), 861-870.

Collado, C. F., Lucio, P. B., \& Sampieri, R. H. (2013). Metodologia de pesquisa. Editora Penso.

Conde, S. V., da Silva, T. N., Gonzalez, C., Carmo, M. M., Monteiro, E. C., \& Guarino, M. P. (2012). Chronic caffeine intake decreases circulating catecholamines and prevents diet-induced insulin resistance and hypertension in rats. British Journal of Nutrition, 107(1), 86-95.

Consumo mundial de café em 2019 atinge 168 milhões de sacas. (n.d.). Retrieved from https://www.embrapa.br/busca-de-noticias//noticia/48512453/consumo-mundial-de-cafe-em-2019-atinge-168-milhoes-de-sacas

Da Costa Santos, V. B., Ruiz, R. J., Vettorato, E. D., Nakamura, F. Y., Juliani, L. C., Polito, M. D., \& de Paula Ramos, S. (2011). Effects of chronic caffeine intake and low-intensity exercise on skeletal muscle of Wistar rats. Experimental physiology, 96(11), 1228-1238.

De Oliveira Mattos, F., de Salles Painelli, V., Gualano, B., \& Lancha Jr, A. H. (2014). Eficácia ergogênica da suplementação de cafeína sobre o desempenho de força? Uma análise crítica. Journal of Physical Education, 25(3), 501-511.

Devenney, S., Mangan, S., Shortall, M., \& Collins, K. (2018). Effects of carbohydrate mouth rinse and caffeine on high-intensity interval running in a fed state. Applied Physiology, Nutrition, and Metabolism, 43(5), 517-521.

Dos Santos, J. E. T., \& Rocha, J. B. T. (1994). Tolerance to methylxanthines and interaction with Dopaminergic System clinical implications. Ciência $e$ Natura, 16(16), 113-124.

Ferreira, G. A., Felippe, L. C., Bertuzzi, R., Bishop, D. J., Ramos, I. S., De-Oliveira, F. R., \& Lima-Silva, A. E. (2019). Does caffeine ingestion before a shortterm sprint interval training promote body fat loss? Brazilian Journal of Medical and Biological Research, 52(12).

Ferreira, G. A., Felippe, L. C., Bertuzzi, R., Bishop, D. J., Barreto, E., De-Oliveira, F. R., \& Lima-Silva, A. E. (2018). The effects of acute and chronic sprintinterval training on cytokine responses are independent of prior caffeine intake. Frontiers in physiology, 9, 671.

Gomes, C. B., de Sá Barreto, A. F. C., Almeida, M. M., Mello, A. O. T., Ide, B. N., \& dos Santos, C. P. C. (2014). Uso de suplementos termogênicos à base de cafeína e fatores associados a qualidade de vida relacionada à saúde em praticantes de atividade física. Revista Brasileira de Prescrição e Fisiologia do Exercício (RBPFEX), 8(49), 695-704.

Guerra, R. O., Bernardo, G. C., \& Gutiérrez, C. V. (2000). Cafeína e esporte. Revista Brasileira de Medicina do Esporte, 6(2), 60-62.

Guest, N., Corey, P., Vescovi, J., \& El-Sohemy, A. (2018). Caffeine, CYP1A2 genotype, and endurance performance in athletes. Medicine \& Science in Sports \& Exercise, 50(8), 1570-1578.

Kasper, A. M., Cocking, S., Cockayne, M., Barnard, M., Tench, J., Parker, L., \& Morton, J. P. (2016). Carbohydrate mouth rinse and caffeine improves highintensity interval running capacity when carbohydrate restricted. European journal of sport science, 16(5), 560-568.

Machado, A. F. (2017). HIIT: manual prático. Phorte Editora LTDA.

Machado, A. F., Evangelista, A. L., Miranda, J. M. Q., Teixeira, C. V., Rica, R. L., Lopes, C. R., \& Bocalini, D. S. (2018). Description of training loads using whole-body exercise during high-intensity interval training. clinics, 73.

Machado, A. F., Evangelista, A. L., Miranda, J. M. D. Q., Teixeira, C. V. L. S., Leite, G. D. S., Rica, R. L., \& Bocalini, D. S. (2018). Sweat rate measurements after high intensity interval training using body weight. Revista Brasileira de Medicina do Esporte, 24(3), $197-201$.

Machado, A. F., Baker, J. S., Figueira Junior, A. J., \& Bocalini, D. S. (2019). High-intensity interval training using whole-body exercises: training recommendations and methodological overview. Clinical physiology and functional imaging, 39(6), 378-383.

Mohr, M., Nielsen, J. J., \& Bangsbo, J. (2011). Caffeine intake improves intense intermittent exercise performance and reduces muscle interstitial potassium accumulation. Journal of applied physiology, 111(5), 1372-1379.

Mundt, M. P., \& Bertolini, E. L. (2016). Eficiência do HIIT comparado ao exercício moderado contínuo voltado ao emagrecimento. Anais do EVINCIUniBrasil, 2(1), 101-101.

Nehlig, A. (2016). Effects of coffee/caffeine on brain health and disease: What should I tell my patients? Practical neurology, 16(2), 89-95.

Santos, A. L. P., Santos, C. O., Rosa, N. R., Souza, P., \& Mazeto, T. K. (2015). Efeito da cafeína no organismo. Rev. Saberes, Rolim de Moura, 3 , 45-52.

Silveira, L. R., Alves, A. A., \& Denadai, B. S. (2008). Efeito da lipólise induzida pela cafeína na performance e no metabolismo de glicose durante o exercício intermitente. Revista Brasileira de Ciência e Movimento, 12(3), 21-26.

Sousa, T. C. D. (2018). Efeitos agudos do high intensity interval training (hiit) sobre o metabolismo glicolítico e oxidativo na corrida em diferentes tipos de recuperação.

Sutton-Tyrrell, K., Wildman, R. P., Matthews, K. A., Chae, C., Lasley, B. L., Brockwell, S., \& Torréns, J. I. (2005). Sex hormone--binding globulin and the free androgen index are related to cardiovascular risk factors in multiethnic premenopausal and perimenopausal women enrolled in the Study of Women Across the Nation (SWAN). Circulation, 111(10), 1242-1249.

Tanno, A. P., \& Marcondes, F. K. (2002). Estresse, ciclo reprodutivo e sensibilidade cardíaca às catecolaminas. Revista Brasileira de Ciências Farmacêuticas, 38(3), 273-289. 